\title{
Detection of bone mineral density changes by subtraction of cone-beam computed tomography images: a pilot study
}

\begin{abstract}
The objective in this study was to investigate the feasibility of digital subtraction technique using cone beam computed tomography (CBCT) images to assess mineral density changes in dry mandibular bone tissue. A simulated procedure to mimic periapical bone loss was performed in 12 alveolar sockets of a dry mandible by controlled application of $0.1 \mathrm{ml}$ of a $70 \%$ perchloric acid solution. Digital periapical radiographs and CBCT exams were taken at baseline and after 2, 4 and 24hours. Simulated mineral gain was also performed in four alveolar sockets using a bone substitute in differing amounts (baseline, 0.07 and $0.14 \mathrm{~g}$ ) aggregated in a wax medium. All images were subtracted from baseline, resulting in four subtracted images $\left(\mathrm{S}_{0}-\mathrm{S}_{1}-\mathrm{S}_{2}-\mathrm{S}_{3}\right)$ for simulated bone loss and three subtracted images for mineral gain $\left(\mathrm{S}_{0}-\mathrm{S}_{1}-\mathrm{S}_{2}\right)$ from periapical radiographs and CBCT. Quantitative analysis of mineral density was performed to assess pixel value changes in a grey scale ranging from 0 (black) to 255 (white) pixels. A gradual decrease in pixel values from $\mathrm{S}_{0}$ to $\mathrm{S}_{3}$ was observed in all images $(\mathrm{p}<0.001)$. Pairwise comparison between periapical radiographs and CBCT slices showed no significant differences, although the subtle changes in the $\mathrm{S}_{1}$ images (after 2hours) were less apparent in the periapical radiographs. A continuous progressive gain in mineral bone was also observed from baseline to $S_{1}$ and $S_{2}$. The results suggest that subtracted CBCT images can be used to monitor mineral bone changes based on the pixel grey values of images, with potential application for evaluation of bone repair.
\end{abstract}

Keywords: subtraction technique, cone--beam computed tomography, bone repair
Volume 8 Issue 5 - 2017

\author{
Thiago Oliveira Sousa,' Claudio Rodrigues \\ Leles, ${ }^{2}$ Elismauro Francisco Mendonca' \\ 'Department of Stomatology (Oral Pathology), Federal \\ University of Goias, Brazil \\ ${ }^{2}$ Department of Prevention and Oral Rehabilitation, Federal \\ University of Goias, Brazil
}

\begin{abstract}
Correspondence: Thiago Oliveira Sousa, Department of Stomatology (Oral Pathology), School of Dentistry, Federal University of Goias, Goiania, Brazil,

Email thiagoodonto@hotmail.com
\end{abstract}

Received: October 17, 2017 | Published: October 23, 2017

\section{Introduction}

Bone repair is expected to occur as a consequence of treating bony lesions and after surgical procedures that result in different degrees of bone injury. This is a dynamic inflammatory process that involves both mineral bone loss and gain, resulting in continuous bone remodelling at the cellular level. This process consists in the removal of mineralized bone by osteoclasts followed by formation of new bone matrix through osteoblasts that subsequently become mineralized..$^{1-16}$ Subtle mineral bone changes are difficult to observe by visual analysis, thus changes in bone are usually monitored using sequential images taken by conventional radiograph methods or computerized tomography at different follow-up periods. Other alternative method to monitor bone healing is the utilization of digital subtraction radiography (DSR) associated to computer-assisted image processing. DSR has been used both in vitroand in clinical studies as a sensitive tool to monitor minor bone changes, including mineral loss and gain..$^{9-18}$ The DSR method is based on the digital alignment and superimposition of two radiographs of the same region obtained at different time. ${ }^{19}$ A subtracted image is produced using a specific software system algorithm that converts the two radiographic images into a homogeneous grey background of 128 pixels. Unchanged regions present grey pixel values close to 128 , while higher values indicate mineral density gain and lower values are compatible with density loss. ${ }^{17-20}$ DSR is commonly used for different purposes in dentistry and subtracted images can be obtained from conventional or digital radiographs. These two-dimensional images of three-dimensional structures can provide only limited information about a region of interest, due to superposition, inherent to bidimensional radiographs, which leads to a limited three-dimensional visualization at different depth levels. This fact has been a limitation of the routine use of subtraction technique in the clinical practice, once the evaluation of volumetric differences in three dimensions would be the ideal when monitoring bone repair. The use of CBCT allows three-dimensional multiplanar sectional imaging and is increasingly used in dentistry due to its advantages such as the relatively low radiation dose, high-resolution images, technical simplicity, and the good visualization of hard tissues. ${ }^{15-22}$ However, subtraction technique has not been applied regularly to CBCT images in order to quantify mineral density changes. This new application would potentially increase the use of the subtraction technique by being associated with the higher image resolution provided by $\mathrm{CBCT}$ scans. Hence, the aim of this study was to investigate the feasibility of applying DSR to sectional images obtained by CBCT in order to evaluate changes in bone density by means of pixel value quantification.

\section{Materials and methods}

Following local ethics committee approval (protocol number 225/12) a dried edentulous mandible was used. The regions of interest (ROI) corresponded to sixteen alveolar sockets with no teeth and presenting an uninjured bone surface. Two experimental assays were tested: 
1. Induction of mineral density loss in periapical region.

2. Induction of mineral density gain in alveolar sockets.

Four alveolar sockets (premolars) were used for induction of mineral gain and the remaining 12 alveolar sockets (molars, canines and incisors) were used to induce bone loss in the apical region. Digital periapical radiographs and $\mathrm{CBCT}$ images were obtained according to the experimental conditions of the study.

\section{Induction of mineral density loss}

After the baseline periapical radiography and CBCT were taken $\left(\mathrm{T}_{0}\right)$, mineral density loss was induced by applying $70 \%$ perchloric acid on a cotton pellet inserted into each of the 12 alveolar sockets containing $0.1 \mathrm{~mL}$ of the acid. ${ }^{14}$ Subsequently, the alveolar sockets were sealed with wax. After 2 hours $\left(\mathrm{T}_{1}\right)$, the pellet was removed, the alveolar sockets were thoroughly washed with distilled water and new radiographic and $\mathrm{CBCT}$ acquisitions were taken. The same procedures were repeated after $4\left(\mathrm{~T}_{2}\right)$ and 24 hours $\left(\mathrm{T}_{3}\right)$. Different times were chosen to test subtle density changes $\left(T_{1}\right.$ and $\left.T_{2}\right)$ and the maximum bone loss possible $\left(\mathrm{T}_{3}\right)$. Duplicate acquisitions were obtained at baseline in order to verify the reliability of the subtraction technique.

\section{Induction of mineral density gain}

In order to simulate mineral density gain, the alveolar sockets were initially filled with wax (baseline). In the subsequent image acquisitions, the alveolar sockets were filled with a biosynthetic bone substitute (composite osseous graft of bovine origin) (GenMix, Baumer, Mogi Mirim, São Paulo, Brazil) aggregated in wax. The grain size of the bone substitute ranged from 0.25 to $1.0 \mathrm{~mm}$, dosed in different quantities weighed on an electronic precision balance (BD-300, Instrutherm, São Paulo, Brazil). Each quantity of bone was mixed with a melted wax matrix and added to each alveolar socket after complete removal of the wax of the first acquisition (baseline). Differently than in the loss induction, in which the alveolar sockets could lose mineral gradually, in the induction of mineral density gain, they were then classified according to the bone substitute quantity in grams: baseline (wax only-Q0), 0.07g (Q1) and 0.14g (Q2).

\section{Radiographic and tomographic acquisitions}

All radiographic images were taken using a periapical $\mathrm{x}$-ray device (Focus Cone 50540, Instrumentarium Dental, Tuusula, Etelä-Suomi, Finland), $70 \mathrm{kVp}, 7 \mathrm{~mA}, 0.16 \mathrm{~s}$. A digital phosphor plate system (Dürr Dental, Bietgheim-Bissingen, Ludwigsburg, German) was used for visualization, with sensors (dimensions $3 \times 4 \mathrm{~cm}$ ) positioned within a radiography holder kit (Indusbello, Indústria de Instrumentos Odontológicos Ltda., Londrina, Paraná, Brazil) and a polyvinyl siloxane guide (Re'Cord, Harry J. Bosworth Company, Skokie Illinois, USA) to ensure geometric reproducibility. Image plates were scanned immediately after exposure and saved as uncompressed TIFF format. The CBCT images were acquired using the $\mathrm{i}-\mathrm{CAT}{ }^{\circledR}$ unity (Imaging Sciences International, Hatfield, Pensylvannia, USA) set with the following protocol: $6 \mathrm{~cm}$ field of view, $0.25 \mathrm{~mm}$ voxel size and $40 \mathrm{~s}$, tube voltage $120 \mathrm{KVp}$ and tube current $3.8 \mathrm{~mA}$. For reliable spatial positioning of the mandible, an acrylic resin base was constructed to allow it to fit into a plastic container. In order to simulate soft tissue and the influence of the scatter radiation, the plastic container was filled with distilled water, ${ }^{6-13}$ and placed at the base of the CBCT device guided by a base made of gypsum (Figure 1).

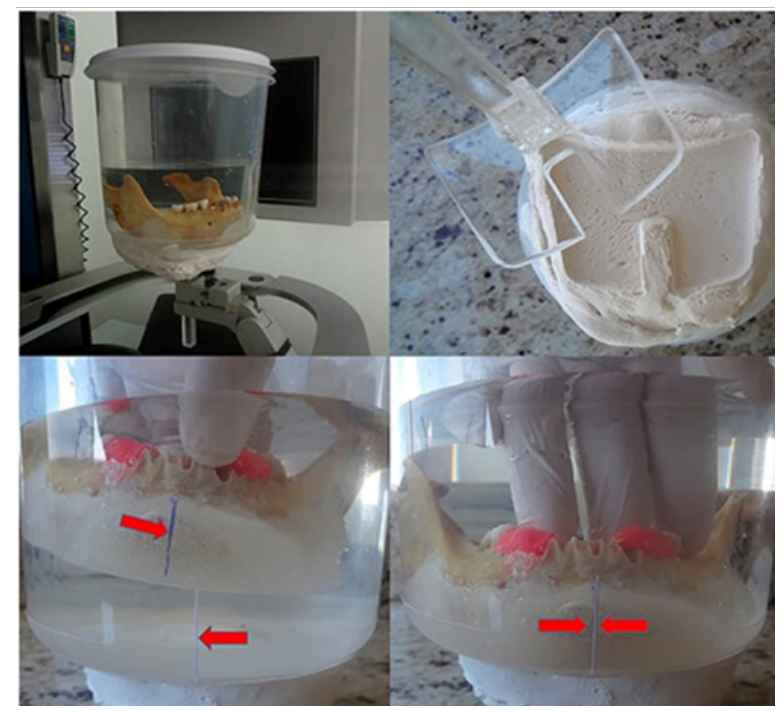

Figure I Spatial stabilization of the mandible with acrylic resin, inside a container filled with distilled water and the detail of the gypsum base, used to stabilize the mandible and permit the same geometrical position. The lower images show how the acrylic base was aligned at the same position.

\section{Image subtraction and quantitative analysis}

In order to detect loss or gain in mineral density in radiographs of chemically created but visually undetectable periapical lesions, the follow-up images were subtracted from their respective baseline image. For mineral density loss, the subtraction procedure was as follows: $\mathrm{T}_{0}-\mathrm{T}_{0}, \mathrm{~T}_{1}-\mathrm{T}_{0}, \mathrm{~T}_{2}-\mathrm{T}_{0}$ and $\mathrm{T}_{3}-\mathrm{T}_{0}$, resulting in four groups of subtracted images $\left(\mathrm{S}_{0}, \mathrm{~S}_{1}, \mathrm{~S}_{2}\right.$ and $\left.\mathrm{S}_{3}\right)$. The ROI corresponded to the central region immediately below the periapical space in the apex of each alveolar socket, $1 \mathrm{~mm}$ below the periodontal cortical bone. Mean pixel values were measured in a $10 \times 10$ pixel area in the central part of the induced lesion as established in previous studies. ${ }^{17,18}$ For mineral density gain, subtraction was performed as follows: Q1-Q0, Q2-Q0. The 10x10pixel region of interest (ROI) was measured in the central portion of each of the three thirds of the alveolar socket (cervical, central and apical). All the digital periapical radiographic images were saved in tagged image file format (TIFF) 8 -bit. The tomographic images were saved in DICOM format and opened using Image $(\mathbb{B}$ software (ImageJ 1.37v, National Institute of Health, Bethesda, MD, USA). The most representative slices (central slice in each alveolar socket) from each previously determined region were selected and also saved in TIFF 8-bit format in three projections: axial, coronal and sagittal. The slice number was identified on Image ${ }^{\circledR}$ software and immediately registered. For the subsequent images of the same region, the volume was scrolled to the registered coordinates, to guarantee slices from the same region and in the respective projection. The Regeemy Image Registration and Mosaicking software (version 0.2.43-RCB DPI-INPE, São José dos Campos, São Paulo, Brazil) was used to perform the digital subtraction of the images. The software system algorithm converts unchanged pre-and post-treatment images into a homogeneous grey background of 128pixels (subtracted image). Assessment of changes in the subtracted images permits a quantitative analysis of bone changes using pixel values in a grey scale ranging from 0 (white) to 255 (black). A cut-off value of 128pixels, located midway between white and black, which discriminates mineral bone 
density loss $(<128$ pixels $)$ and gain $\left(<128\right.$ pixels). ${ }^{17,18}$ The software enables gamma correction in order to compensate for small changes in contrast and density, as well as anatomical alignment of the two images. ${ }^{23}$ The selection of the points was made based on anatomic marks (Figure 2). After subtraction, the subtracted images were also saved in the TIFF format (8-bit). For analysis of subtle changes in mineral bone density the histogram tool in the software was used to provide a quantitative measure of the pixel grey values in each region of interest ROI, considering a grey scale of pixels. The X/Y coordinates were used to reproduce the same position of the ROI in all subtracted images. All the images were analysed by one experienced Radiologist, previously calibrated. Figures $3 \& 4$ illustrate the image sequence for assessing changes in pixel grey values in the areas of simulated mineral density loss and gain, respectively, using CBCT subtracted images. Mineral density variation on the radiographs can be observed in the Figures $5 \& 6$.

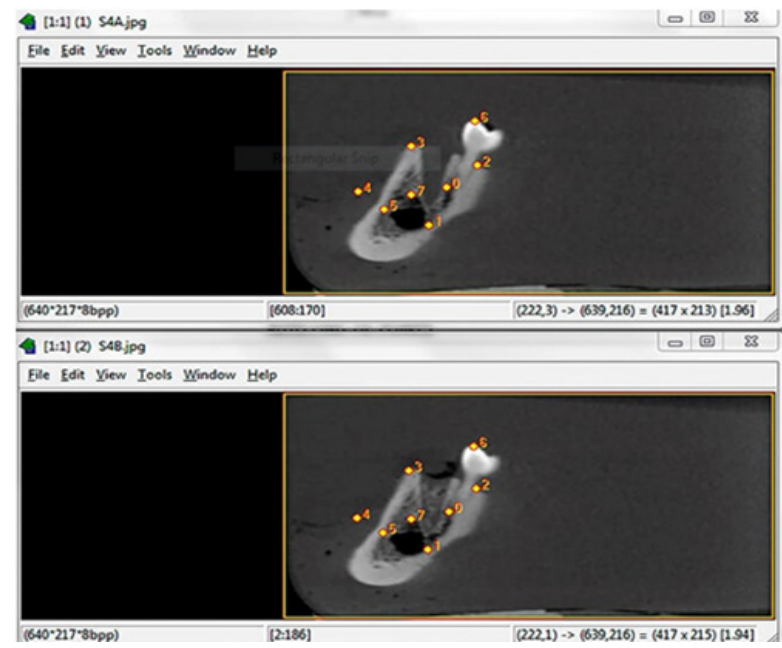

Figure 2 Screen capture of Regeemy Image Registration and Mosaicking software showing the automatic alignment process of 2 sagittal images from the same alveolar socket, which is based in landmarks precisely detected and confirmed numerically by the software. The upper slice shows an alveolar socket filled with wax only and the lower a Q2 concentration.

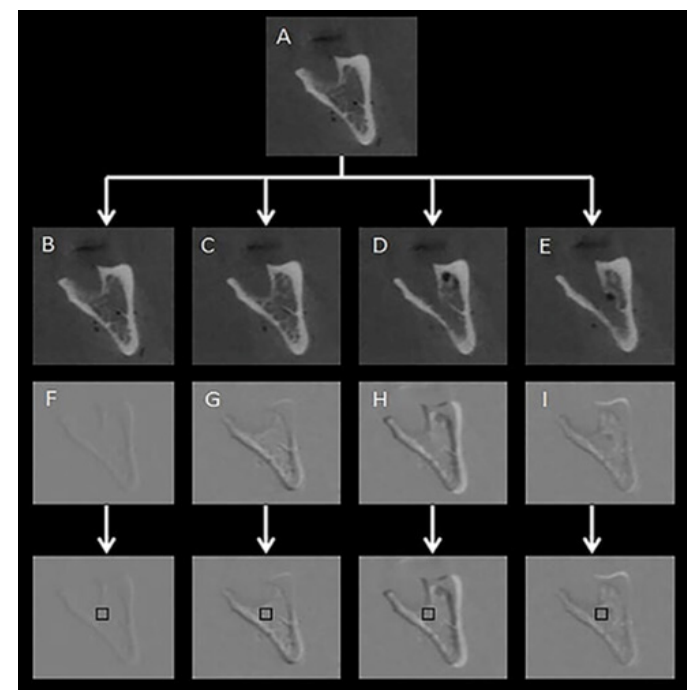

Figure 3 Coronal slice through the alveolar socket of the left second molar. $\mathrm{A}$ : Sequence of CBCT images of simulated periapical bone loss by controlled application of $0.1 \mathrm{ml}$ of a $70 \%$ perchloric acid solution at baseline; B: after 2 , 4 and 24hours (C to E). The subsequent subtracted images $S_{0}(F), S_{1}(G), S_{2}$ $(\mathrm{H})$ and $\mathrm{S}_{3}(\mathrm{l})$ and the corresponding ROls for quantitative pixel grey value evaluation are also presented.

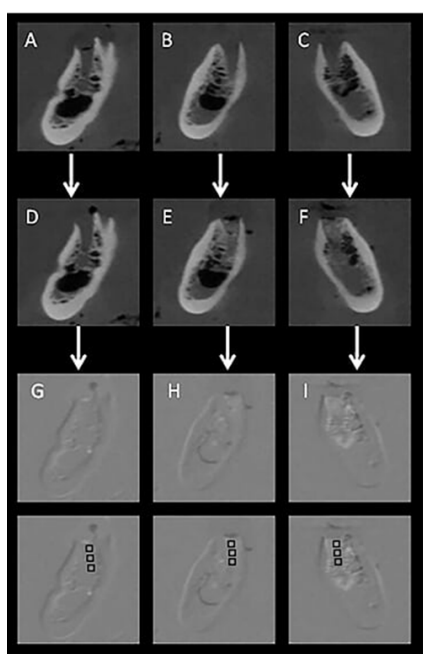

Figure 4 Coronal CBCT images of 3 different alveolar sockets (premolar regions from the same specimen) showing the progressive mineral gain in terms of pixel grey values from baseline $(A, B, C)$ and after filling with different quantities of bone substitute: $D$ : baseline-no filling; $\mathrm{E}: 0.07 \mathrm{~g} ; \mathrm{F}: 0.14 \mathrm{~g}$, followed by the respective subtracted images; $\mathrm{G}, \mathrm{H} \& \mathrm{I}$ : ROI schemes for measuring the increase in mineral density in the center of each third of the alveolar socket (cervical, central and apical).

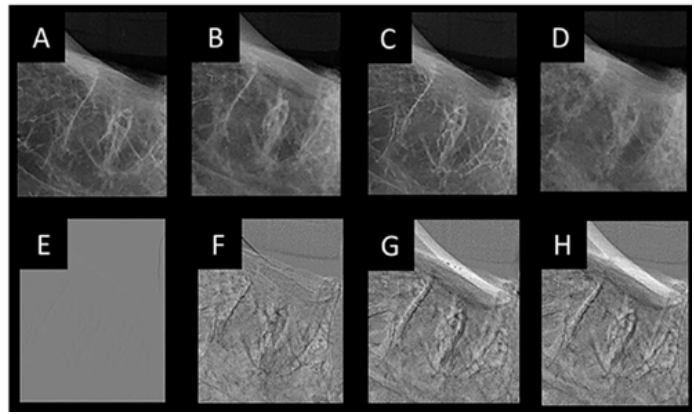

Figure 5 Socket in the lower molar region showing decrease in mineral density along time: A: baseline; B: 2 hours; C: 4 hours; D: 24 hours. E, F, G and H correspond to the images of $S_{0}, S_{1}, S_{2}$ and $S_{3}$ of the same region.

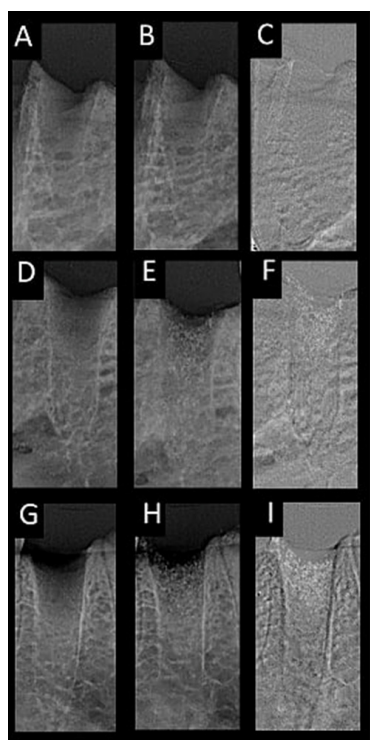

Figure 6 Radiographs acquired in the simulated mineral gain, showing the sockets filled with wax (A, D and $G$ ) in the first acquisition; Q0, QI and Q2 in the second acquisition (B, E and $\mathrm{H}$, respectively); and the subtracted images from their correspondent regions ( $\mathrm{C}, \mathrm{F}$ and $\mathrm{I})$. 


\section{Data analysis}

MedCalc Software version 12.4.0 (MedCalc Software, Ostend, Belgium) was used for statistical analysis. Data are expressed as the mean and standard deviation of the pixel values and the confidence interval was set at $95 \%$. The subtracted digital periapical images (periapical digital radiography) were considered as the reference method for comparison with CBCT subtracted images (CBCT axial, coronal and sagittal slices). The comparison of different types of images and different subtracted images $\left(\mathrm{S}_{0}, \mathrm{~S}_{1}, \mathrm{~S}_{2}, \mathrm{~S}_{3}\right)$ for simulated bone loss was performed using repeated-measures ANOVA to test within-group and between-groups effects. The Bonferroni test was used for between-groups contrasts. The level of significance for rejecting the null hypothesis was 0.05 .

\section{Results}

There were marked changes in both mineral loss and gain under the tested experimental conditions. Figure 7 shows the measures of pixel grey values in the experimental mineral density loss test, according to the subtracted images $\left(\mathrm{S}_{0}\right.$ to $\left.\mathrm{S}_{3}\right)$ in periapical radiography and CBCT images in sagittal, axial and coronal slices. There was a significant decrease in mineral density (from $\mathrm{S}_{0}$ to $\mathrm{S}_{3}$ ) for the subtracted radiographs and CBCT scans $(\mathrm{p}<0.001)$. The image versus method interaction was also statistically significant $(\mathrm{p}=0.003)$. The betweengroups comparison $(\mathrm{p}=0.023)$ showed significant difference between the subtracted radiograph and the coronal slice (mean difference $=5.87$ pixels; $\mathrm{p}=0.033$ ). Figure 8 depicts the values of the induction of mineral density gain in pixel grey values measured in the subtracted images $\left(\mathrm{S}_{0}, \mathrm{~S}_{1}\right.$ and $\left.\mathrm{S}_{2}\right)$, according to the image type and the bone substitute quantity in grams (Q0, Q1 and Q2). In all imaging methods there was an increase in mineral density in Q1 and Q2 compared to the homogeneous grey background of 128 pixels (Q0). Higher Q1 and Q2 values were observed in the axial and coronal CBCT scans.
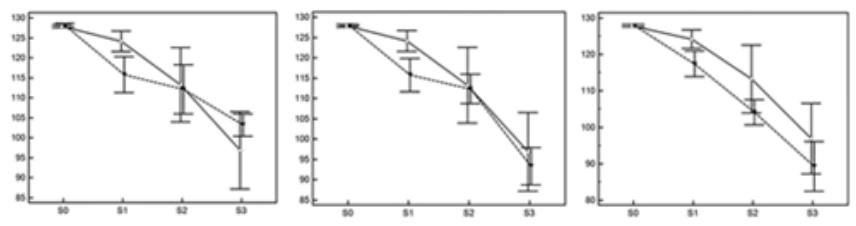

Figure 7 Mean and $95 \% \mathrm{Cl}$ of pixel grey values in the simulation of minera density loss according to the subtracted images $\left(\mathrm{S}_{0}\right.$ to $\left.\mathrm{S}_{3}\right)$. The solid lines represent the periapical radiographic image values and the dashed lines represent the CBCT measurements for sagittal (left), axial (centre) and coronal (right) slices.

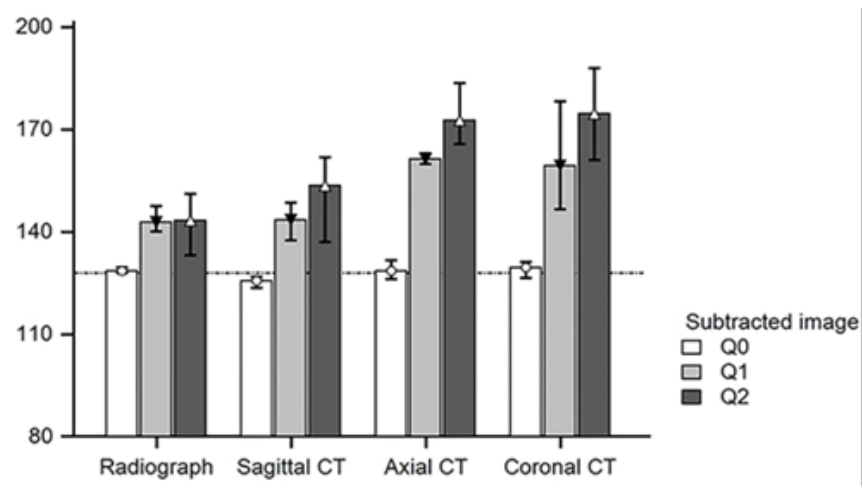

Figure 8 Mean and range (min-max) of pixel grey values for simulated mineral density gain according to the type of image and the subtracted image $\left(\mathrm{S}_{0}, \mathrm{~S}_{1}, \mathrm{~S}_{2}\right)$ The horizontal reference line is I 28 pixels (homogeneous grey background).

\section{Discussion}

Several in vitro and in vivo studies have focused on monitoring bone healing. However, to the best of the authors' knowledge, this is the first study to assess pixel values from sectional images from CBCT obtained by DSR. Our results show that experimentally induced mineral density changes can be quantitatively measured using DSR under controlled conditions. Earlier studies have shown that chemically induced lesions can be detected in periapical images, even in the case of short-term acid exposure. ${ }^{7-14}$ Our study proposed a method that permitted the detection of the progression of incipient lesions with subtle mineral loss, as well as subtle changes in mineral bone gain that would be detectable in remodelling bone repair process. These slight changes, which could be hardly detected in visual analysis by the naked eye, ${ }^{24}$ were identified and quantitatively measured by means of pixel grey values using both CBCT and digital periapical images. The patterns of mineral changes detected in both CBCT and periapical images were similar, which may be justified by the fact that CBCT is a type of exam that produces minimal distortion in the final image. ${ }^{25}$ and is a suitable option for analysing bone mass, being as feasible as periapical radiographs for the DSR technique. ${ }^{17,18}$ Economomopoulos et al. ${ }^{26}$ presented a method for automatically aligning 3D CBCT dental data sets. This proposed method was able to evaluate volumetric differences between data sets. In our study it was possible to detect subtle changes in bone density at the periapical region using quantitative pixel measurement in CBCT images, and periapical radiography as reference method. In this study, when analysing the decrease in pixel values throughout the sequential subtractions, CBCT values were slightly lower than those from periapical radiographs, possibly due to the fact that conventional digital radiographic images show bone structures overlapping (sound and injured bone). Considering that the $\mathrm{CBCT}$ slices were obtained from the central regions of alveolar sockets (coronal and sagittal) and immediately below (in axial slices), the second image tended to record the central portion of the lesion in $0.25 \mathrm{~mm}$ thick slices, with no superimposition of intact bone and consequent lowering of the pixel grey values. This feature is in accordance with Sogur et al, ${ }^{27}$ who compared $\mathrm{CBCT}$ and periapical (conventional and phosphor plate system) images in order to investigate the detectability of chemically induced lesions. They concluded that a larger volume of bone loss is required before periapical radiolucency becomes evident on a radiographic film or digital periapical image. The homogeneity in pixel grey values at baseline (for both bone gain and loss) occurred because the initial subtracted image was obtained from specimens without any bone substitute or acid exposition. Conversely, greater variability was observed in the subsequent subtracted images. Haiter-Neto \& Wenzel. ${ }^{2}$ pointed out that the more shades of grey there are in a histogram, the more noise there will be in the subtracted radiographic image and the larger the standard deviation. Hence, the highest variability in the values in induced bone loss were found in groups of radiographs, probably due to the size of the images: a single radiographic image has greater resolution than a CBCT slice and, consequently, a greater number of pixels and a larger range of pixel values. Bornstein et al. ${ }^{28}$ compared the efficacy of periapical radiography and $\mathrm{CBCT}$ imaging in detecting periapical lesions in mandibular molars referred for apical surgery. The study showed that $25.9 \%$ of lesions detected by sagittal CBCT sections were not detected by periapical radiography. It was also concluded that periapical radiography underestimates the true extent of lesions compared with coronal and sagittal CBTC slices. Estrela et al. ${ }^{29}$ also observed that $\mathrm{CBCT}$ was a more reliable diagnostic method to identify apical periodontitis. Accuracy analysis showed that periapical lesions were correctly identified in $54.5 \%$ of 
the cases with periapical radiographs and in $27.8 \%$ of the cases with panoramic radiographs; in most cases, they were correctly identified using conventional methods only when in an advanced stage. In order to obtain a grey scale in CBCT, several studies. ${ }^{9-14}$ have tried to correlate. ${ }^{4-11}$ or derive. ${ }^{2-30}$ Hounsfield Units (HU) values in CBCT exams. Considering the larger amount of scattered $\mathrm{X}$-rays in CBCT compared to $\mathrm{CBCT}$ acquisitions, it was stated that $\mathrm{HU}$ values in $\mathrm{CBCT}$ cannot be considered a valid method for quantifying mineral density. ${ }^{31}$ In our study it was not used a large grey scale such as the HU scale. An 8-bit scale was used instead, which means that all images were converted into a smaller range pixel gray scale, containing 256 shades of grey, ranging from 0 (black) to 255 (white). ${ }^{28}$ However, even not using HU measurements, it is important to consider the instability of grey values when dealing with CBCT acquisitions. Nevertheless, we found a satisfactory correspondence for measurements of mineral bone changes when converting the images into 8-bit system images from both CBCT exams and periapical radiographs. The improved diagnostic performance of CBCT exams observed in clinical studies emphasizes the relevance of exploring different strategies using 3-dimensional evaluation with the DSR technique. DSR has already been reported previously in the context of bidimensional examinations, being considered a non-invasive method to evaluate longitudinal mineral changes. ${ }^{17-32}$ For patients with extensive bone lesions in which bone repair is expected to occur, CBCT is frequently used as a tool in monitoring bone healing and new methodology has been proposed to obtain a satisfactory alignment between structure to be monitored..$^{27-33}$ however, more studies are needed to strengthen the use of DSR in CBCT images. There is no doubt that visual image analysis presents some disadvantages like the inability to detect initial bone loss. ${ }^{19}$ and misinterpretation due to the subjectivity inherent in image interpretation, ${ }^{7-12}$ which can be overcome by an objective quantitative assessment using pixel grey values.

\section{Conclusion}

In conclusion, this exploratory in vitro study showed promising results concerning the quantification of pixel values during bone repair processes using DSR on CBCT sectional images. However, although this pilot study provided support for new studies using subtracted CBCT images to monitor bone repair in clinical situations, it is important to recognize that the results of in vitro studies cannot be extrapolated uncritically to in vivo situations, which emphasizes the need for further clinical studies.

\section{Acknowledgments}

None.

\section{Conflicts of interest}

The author declares that there is no conflict of interest.

\section{Funding}

None.

\section{References}

1. Hadjidakis DJ, Androulakis II. Bone remodeling. Ann N Y Acad Sci. 2006;1092:385-396.

2. Haiter-Neto F, Wenzel A. Noise in subtraction images made from pairs of bitewing radiographs: a comparison between two subtraction programs. Dentomaxillofac Radiol. 2005;34(6):357-361.
3. Hohlweg-Majert B, Metzger MC, Kummer T, et al. Morphometric analysis - Cone beam computed tomography to predict bone quality and quantity. J Craniomaxillofac Surg. 2011;39(5):330-334.

4. Lagravère MO, Carey $\mathrm{J}$, Ben-Zvi M, et al. Effect of object location on the density measurement and Hounsfield conversion in a NewTom 3G cone beam computed tomography unit. Dentomaxillofac Radiol. 2008;37(6):305-308.

5. Mah P, Reeves TE, McDavid WD. Deriving Hounsfield units using grey levels in cone beam computed tomography. Dentomaxillofac Radiol. 2010;39(6):323-335.

6. Marques AP, Perrella A, Arita ES, et al. Assessment of simulated mandibular condyle bone lesions by cone beam computed tomography. Braz Oral Res. 2010;24(4):467-474.

7. Meier AW, Brown CE, Miles DA, et al. Interpretation of chemically created periapical lesions using direct digital imaging. J Endod. 1996;22(10):516-520.

8. Miguens SA Jr, Veeck EB, Fontanella VR, et al. A comparison between panoramic digital and digitized images to detect simulated periapical lesions using radiographic subtraction. J Endod. 2008;34(12):1500-1503.

9. Mikrogeorgis G, Lyroudia K, Molyvdas I, et al. Digital radiograph registration and subtraction: a useful tool for the evaluation of the progress of chronic apical periodontitis. J Endod. 2004;30(7):513-517.

10. Nackaerts O, Maes F, Yan H, et al. Analysis of intensity variability in multislice and cone beam computed tomography. Clin Oral Implants Res. 2011;22(8):873-879.

11. Naitoh M, Aimiya H, Hirukawa A, et al. Morphometric analysis of mandibular trabecular bone using cone beam computed tomography: an in vitro study. Int J Oral Maxillofac Implants. 2010;25(6):1093-1098.

12. Nicopoulou-Karayianni K, Bragger U, Patrikiou A, et al. Image processing for enhanced observer agreement in the evaluation of periapical bone changes. Int Endod J. 2002;35(7):615-622.

13. Noujeim M, Prihoda T, Langlais R, et al. Evaluation of high-resolution cone beam computed tomography in the detection of simulated interradicular bone lesions. Dentomaxillofac Radiol. 2009;38(3):156-162.

14. Ozen T, Kamburoglu K, Cebeci AR, et al. Interpretation of chemically created periapical lesions using 2 different dental cone-beam computerized tomography units, an intraoral digital sensor, and conventional film. Oral Surg Oral Med Oral Pathol Oral Radiol Endod. 2009;107(3):426-432.

15. Scarfe WC, Farman AG, Sukovic P. Clinical applications of conebeam computed tomography in dental practice. J Can Dent Assoc. 2006;72(1):75-80.

16. Sezgin Y, Uraz A, Taner IL, et al. Effects of platelet-rich fibrin on healing of intra-bony defects treated with anorganic bovine bone mineral. Braz Oral Res. 2017;31(1):e1-e11.

17. Benfica e Silva JB, Leles CR, Alencar AH, et al. Digital subtraction radiography evaluation of the bone repair process of chronic apical periodontitis after root canal treatment. Int Endod J. 2010;43(8):673-680.

18. Carneiro LS, Da Cunha HA, Leles CR, et al. Digital subtraction radiography evaluation of longitudinal bone density changes around immediate loading implants: a pilot study. Dentomaxillofac Radiol. 2012;41(3):241-247.

19. Gröndahl HG, Gröndahl K, Webber RL. A digital subtraction technique for dental radiography. Oral Surg Oral Med Oral Pathol. 1983;55(1):96-102.

20. Christgau M, Hiller KA, Schmalz G, et al. Quantitative digital subtraction radiography for the determination of small changes in bone thickness: an in vitro study. Oral Surg Oral Med Oral Pathol Oral Radiol Endod. 1998;85(4):462-472. 
21. Alonso MB, Vasconcelos TV, Lopes LJ, et al. Validation of cone-beam computed tomography as a predictor of osteoporosis using the Klemetti classification. Braz Oral Res. 2016;30(1).

22. Carter L, Farman AG, Geist J, et al. American Academy of Oral and Maxillofacial Radiology. American Academy of Oral and Maxillofacial Radiology executive opinion statement on performing and interpreting diagnostic cone beam computed tomography. Oral Surg Oral Med Oral Pathol Oral Radiol Endod. 2008;106(4):561-562.

23. Sunku R, Roopesh R, Kancherla P, et al. Quantitative digital subtraction radiography in the assessment of external apical root resorption induced by orthodontic therapy: a retrospective study. J Contemp Dent Pract. 2011;12(6):422-428.

24. Soğur E, Baksı BG, Gröndahl HG, et al. Pixel intensity and fracta dimension of periapical lesions visually indiscernible in radiographs. $J$ Endod. 2013;39(1):16-19.

25. Wakoh M, Nishikawa K, Otonari T, et al. Digital subtraction technique for evaluation of peri-implant bone change in digital dental imaging. Bull Tokyo Dent Coll. 2006;47(2):57-64.

26. Economopoulos TL, Asvestas PA, Matsopoulos GK, et al. Volumetric difference evaluation of registered three-dimensional pre-operative and post-operative CT dental data. Dentomaxillofac Radiol. 2012;41(4):328-339.
27. Sogur E, Baksi BG, Grondahl HG, et al. Detectability of chemically induced periapical lesions by limited cone beam computed tomography, intra-oral digital and conventional film radiography. Dentomaxillofac Radiol. 2009;38(7):458-464.

28. Bornstein MM, Lauber R, Sendi P, et al. Comparison of periapical radiography and limited cone-beam computed tomography in mandibular molars for analysis of anatomical landmarks before apical surgery. $J$ Endod. 2011;37(2):151-157.

29. Estrela C, Bueno MR, Leles CR, et al. Accuracy of cone beam computed tomography and panoramic and periapical radiography for detection of apical periodontitis. J Endod. 2008;34(3):273-279.

30. Bryant J. Deriving Hounsfield units from the grey scale of a CBCT?. Dentomaxillofac Radiol. 2011;40(1):65.

31. Bilhan $\mathrm{H}$, Arat $\mathrm{S}$, Geckili $\mathrm{O}$. How precise is dental volumetric tomography in the prediction of bone density? Int J Dent. 2012;2012:348908.

32. Du Tré F, Jacobs R, Styven S, et al. Development of a novel digital subtraction technique for detecting subtle changes in jawbone density. Clin Oral Investig. 2006;10(3):235-248.

33. Villoria EM, Lenzi AR, Soares RV, et al. Post-processing open-source software for the CBCT monitoring of periapical lesions healing following endodontic treatment: technical report of two cases. Dentomaxillofac Radiol. 2017;46(1):20160293. 